\title{
UNA MIRADA A LOS MÉTODOS DE VALORACIÓN DE CALIDAD AMBIENTAL DESDE UNA VISIÓN CONTABLE AMBIENTAL
}

José Julio Vergara-Arrieta

Yesith Álvarez-Carval 
Panorama Económico, 24 (Octubre 2016 - Septiembre 2017), pp. 221-238

José Julio Vergara-Arrieta

Yesith Álvarez-Carval

\title{
Una mirada a los métodos de valoración de calidad ambiental desde una visión contable ambiental
}

\begin{abstract}
Resumen
En este documento se realiza una revisión bibliográfica de los principales métodos de valoración económica de Bienes y Servicios Ambientales - BSA, analizando sus bondades, limitantes y armonización con la corriente contable ambiental, y respondiendo a interrogantes como: ¿Por qué de la necesidad de valorar los ecosistemas? y ¿qué papel juega esta corriente en la discusión respecto a la crisis ecológica? Se siguió una metodología basada en la identificación, validación y análisis de fuentes científicas originales, consignadas en fichas de lectura estructuradas para este fin, y la conformación de categorías de análisis. Los resultados muestran que los BSA son susceptibles de valorarse económicamente frente a los imperativos de la teoría económica, donde el método de Valoración Integral se inserta de mejor manera en los principios de la contabilidad, haciendo que la corriente ambiental de esta disciplina, se convierta en herramienta vital en el análisis de costo-beneficio, al incorporar las mediciones y valoraciones de los costos ambientales en la dinámica de toma de decisiones, gracias a su poder instrumental y lógico que facilita la abstracción de las relaciones naturaleza-empresa. Palabras Clave: Valoración Económica Ambiental, Contabilidad Ambiental, Bienes y Servicios Ambientales. Clasificación JEL: Q56, Q57, M41.

\section{Looking at the environmental quality estimation methods from an environmental accounting perspective}

\begin{abstract}
This document presents a bibliographic review about the main methods of economic valuation of Environmental Goods and Services (EGS) analyzing their benefits, their limiting factors and their harmonization with the environmental accounting subset. Throughout this document, we try to answer the next questions: Why is it important to value the ecosystems? What role does this accounting subset play in the discussion about the ecological crisis? To carry out this study, we used a methodology based on the identification, validation and analysis of original scientific sources, recorded in structured reading sheets, and the formation of categories of analysis. The results show that EGSs are susceptible to be valued economically despite the imperatives of the economic theory; we could also see that the comprehensive assessment method is better placed in the accounting principles. In this regard, this environmental accounting subset comes out as a vital tool for the costs and benefits analysis while incorporating measures and valuations of environmental costs into the dynamics of decision-making. Thanks to its instrumental and logical power, this subset facilitates the abstraction of relationships between nature and companies.

Keywords: Environmental accounting, environmental goods and services.
\end{abstract}

JEL Classification: Q56, Q57, M41.

\section{Un regard sur les méthodes d'évaluation de la qualité environnementale d'un point de vue comptable-environnemental}

\section{Résumé}

Ce document présente une analyse bibliographique des principales méthodes d'évaluation économique des Biens et Services Environnementaux (BSE) et aborde leurs avantages, leurs limites et leur harmonisation avec la comptabilité environnementale. À travers ce document, nous essayons de répondre aux questions suivantes: Pourquoi est-il important de valoriser les écosystèmes? Quel est le rôle de cette théorie comptable dans la discussion sur la crise écologique? Dans cette étude, nous avons utilisé une méthodologie basée sur l'identification, la validation et l'analyse de sources scientifiques originales, enregistrées dans des fiches de lecture structurées, et la création de catégories d'analyse. Les résultats montrent que les BSE sont susceptibles d'être évalués économiquement malgré les impératifs de la théorie économique. Nous pouvons constater que la méthode d'évaluation globale est mieux placée dans les principes comptables. À cet égard, la comptabilité environnementale apparaît comme un outil essentiel pour l'analyse des coûts-bénéfices. L'incorporation de mesures et évaluations de coûts environnementaux dans la prise de décisions renforcent son pouvoir instrumental et logique. Cette comptabilité facilite l'abstraction des relations entre la nature et les entreprises. Mots-clés: Évaluation économique environnementale, comptabilité environnementale, Bien et Services Environnementaux.

Nomenclature JEL: Q56, Q57, M41. 


\section{UNA MIRADA A LOS MÉTODOS DE VALORACIÓN DE CALIDAD AMBIENTAL DESDE UNA VISIÓN CONTABLE AMBIENTAL}

INFORMACIÓN DEL ARTÍCULO

Recepción de articulo: 24 de febrero de 2016

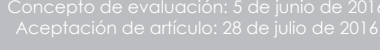

(1)
José Julio Vergara-Arrieta Fundación Universitaria Colombo Internacional Colombia

Yesith Álvarez-Carval Grupo Investigativo de Estudios Ambientales GIDEA

\section{INTRODUCCIÓN}

Desde que el hombre aceptó la finitud de los recursos naturales a su disposición, se han realizado esfuerzos incesantes por establecer parámetros, métodos, metodologías y teorías que buscan establecer un uso sostenible de la naturaleza por parte del mismo, de tal suerte que la cantidad limitada de los recursos sea entendida oportunamente para no causar traumatismos en la economía.

En este sentido las ciencias económicas no han sido ajenas a esta tarea, siendo éstas las encargadas de establecer las formas óptimas de administrar los recursos a disposición del hombre, especialmente los naturales, quienes son los proveedores de los insumos necesarios para la producción empresarial, y también los receptores de los desechos que genera la misma. Por ello nace la Economía Ambiental y la Contabilidad Ambiental, como respuesta a la necesidad de solucionar el problema de valoración de los recursos naturales, que aunque suene absurdo, es una tarea primordial dentro del sentido de conservación de la naturaleza. Así, la valoración de los BSA se concibe como una estrategia para crear conciencia en los individuos, que tales bienes no son gratuitos y que por tanto poseen un valor.

Es así como el presente texto busca analizar los idearios y sustentos teóricos de los principales modelos de valoración económica de la calidad ambiental, desde una perspectiva integradora, sin olvidar que estos modelos constituyen un componente más para el análisis integral que se debe realizar en todo proceso de toma de decisiones, función esencial de la disciplina contable moderna.

${ }^{1}$ Autor para correspondencia. Correo electrónico: jvergara@unitecnologica.edu.co, jovear6@hotmail.com; yealca1991@gmail.com 
En primera instancia se pretende realizar un acercamiento conceptual a la teoría del valor, pretendiendo responder por qué y para qué se valora. Seguidamente se analizarán los postulados y esencia de los métodos de estimación del cambio en productividad, valoración contingente y valoración integral. Finalmente, se abordarán las conclusiones emanadas del análisis del trabajo, las cuales pretenden establecer cuál de los métodos analizados se adapta mejor a la contabilidad ambiental.

\section{METODOLOGÍA}

Para la elaboración del presente artículo se realizó un extenso rastreo bibliográfico con la intención de comprender a fondo los distintos métodos de valoración económica de BSA, y así determinar cuál es el más apropiado en concordancia con los planteamientos de la contabilidad ambiental. En tal sentido, la investigación es de naturaleza cualitativa y descriptiva (Anguera, 1986; Norena, Alcaraz, Rojas y Rebolledo, 2012), en la medida que busca especificar los sustentos teóricos de tales métodos, midiendo y contextualizando sus componentes, bondades y contradicciones, con base en una investigación documental. Dicho análisis, fundado en los planteamientos de Bobenrieth (2002); Dekanski (2014); Avanzas, Bayes-Genis, Pérez, Sanchis y Heras (2011); Páramo, Ortega y Rodríguez (2008); Corral y Encinas (2013); Boyer (2014); Moreno, Marthe y Rebolledo (2010) y Albet (2002), se realizó para la localización, identificación, selección y validación de trabajos científicos en la materia, evaluados a raíz de los planteamientos propuestos por Bobenrieth (1994): claridad, concisión, precisión y sencillez y naturalidad; además, se tuvo en cuenta la trayectoria investigativa de los autores. La búsqueda se realizó en bases de datos científicas como Scopus, ScienceDirect, Ebsco, E-libro, Redalyc, Dialnet, entre otras, así como en diversos libros y revistas indexadas consultadas en físico y digital. El contenido de las fuentes seleccionadas se registró en fichas de lectura construidas y ajustadas a las necesidades informativas de trabajo, que luego servirían para conformar categorías de análisis y síntesis relacionadas en procesos deductivo-inductivos, orientadas a identificar cuál de los métodos analizados es más congruente con la contabilidad ambiental, y qué papel cumple ésta en la discusión académica de la valoración económica ambiental.

\section{RESULTADOS}

En Colombia y en todos los países del mundo cada día es creciente la preocupación por volcarse hacia la competitividad y la internacionalización, por ello se han firmado tratados, entre ellos los de libre comercio, que demandan la mejora significativa de la infraestructura tecnológica, vial, logística, portuaria y urbana. Para la solución de estas necesidades surgen proyectos públicos, privados y/o mixtos que generan impactos significativos en el medio social y ambiental.

Entonces, nace la necesidad de establecer un valor aproximado de BSA que históricamente se han considerado gratuitos, con el ánimo de imprimir el uso y distribución eficiente de estos bienes (Gorfinkiel, 1999; Vergara-Serpa, Navarro, y Cifuentes, 1996). Ante tal situación, las ciencias económicas se han dado a la tarea de crear métodos o técnicas para tal fin, los cuales se analizarán para determinar el más idóneo a la luz de los planteamientos de la contabilidad ambiental, rama de la disciplina contable que ha tomado auge e importancia debido a las actuales problemáticas ambientales, y que al igual que todas las ciencias, está en la búsqueda 
constante de aportes sustanciales al desarrollo del conocimiento científico y, por ende, de alternativas de solución a tales problemáticas (Ortega, 2007). Pero, ¿por qué se establecería el valor de un bien que se considera gratuito? ¿Cómo se establece este valor? ¿Cómo contribuye la contabilidad ambiental al debate que tal temática genera?

Para responder a estos interrogantes se realizará un análisis de los principales métodos de valoración de la calidad ambiental, a pesar de lo intrincado que resulte tal tarea (Ezzine, Rico, Ruiz \& Maris, 2011). Pero antes de abordar estas temáticas, es imperioso conceptualizar el termino valor, viéndolo en un principio desde un enfoque holístico para luego aterrizarlo en el campo ambiental.

Según la Real Academia Española el valor se define valor como "grado de utilidad o aptitud de las cosas, para satisfacer las necesidades o proporcionar bienestar o deleite". Al analizar la definición se evidencia una tendencia a pensar en que las cosas que generan utilidad son las que tienen valor. Por tanto, aquellas que carecen de utilidad inmediatamente carecen de valor. Está demás advertir que la utilidad a que se refiere la definición está íntimamente ligada al servicio del hombre, y no de otros seres de la naturaleza como animales y plantas.

Si se habla del medio ambiente como un conjunto de recursos disponibles a la voluntad humana, incluso por mandato divino ${ }^{1}$, que es parte activa de la economía creada por el hombre para la satisfacción de sus necesidades vitales, entonces se evidencia la utilidad que este tiene para

1 Esto se encuentra explícito en la Biblia, libro del Génesis capítulo 1 versículos 26 al 30 (Sagrada Biblia Católica). el ser humano. Por tanto, a la luz de la anterior definición, se infiere que el medio ambiente es susceptible de ser valorado.

Ahora bien, surge el siguiente interrogante: ¿Desde qué perspectiva es preciso y oportuno realizar la valoración de la calidad de los recursos ambientales? Para responder a este cuestionamiento se traerá a colación las ideas primigenias del liberalismo económico. Para Adam Smith (1776) el valor de un bien o servicio, está dado por la cantidad que lleva de trabajo incorporado. La teoría del valor nace por la relación entre una necesidad humana $\mathrm{y}$ un bien o servicio que la satisfaga. Esto quiere decir que el valor siempre será subjetivo debido a que depende ciento por ciento de la necesidad que busque satisfacer el ser humano.

Desde esta perspectiva, meramente economicista y primigenia, el ser humano no podría darle valor a los recursos naturales dado que éstos no llevan incorporado ningún trabajo antrópico. En este orden de ideas, y dado que para Smith y muchos otros economistas clásicos los recursos naturales eran infinitos, la postura contrasta con la idea de darles un valor a los mismos. Incluso, Crocker citado por Gómez-Baggethun (2011) plantea que "más allá de las breves observaciones de John Stuart Mill² [...], ningún economista de peso deliberó sobre los servicios ambientales y otros beneficios generados por la naturaleza".

"Asimismo, David Ricardo desautorizaba la contribución de los servicios de los ecosistemas a la conformación de valor de cambio cuando señalaba que 'los agentes naturales nos benefician [...] mediante su contribución al valor de uso, pero al actuar

2 Mill (1848) planteó augurios sobre la inevitabilidad (y deseabilidad) de una tendencia de la economía hacia el estado estacionario (Gómez-Baggethun, 2011). 
de forma gratuita, al no ser necesario tener que pagar por el uso del aire, el calor del sol o el agua, la ayuda que nos prestan no aporta nada al valor de cambio'. Al igual, William Petty, señaló que 'la tierra es la madre del valor y el trabajo es el padre'; Karl Marx consideraba que el valor era fruto de la acción conjunta ser humano-naturaleza". (Gómez-Baggethun, 2011).

Para los economistas modernos, que entendieron la limitación de los recursos naturales, el valor de los mismos, visto sólo desde un enfoque economicista, debe responder a las necesidades deinformación y toma de decisiones de la economía de mercado. Por tanto, para las teorías económicas modernas es concebible la idea de asignar valor a un bien ambiental, de tal manera que la Economía Ambiental cumple con el objetivo de teorizar tales aspectos para darles un carácter científico (Reynaldo, 2012). Esto no quiere decir que existe una respuesta definitiva a la problemática de la valoración económica del medio ambiente, más aún, los retos para la ciencias económicas siguen en pie (Gómez, 2005), esencialmente en la estimación de valor de cambio de servicios ambientales (Ezzine et al., 2011; GómezBaggethun, 2011).

La teoría de valor también enfatiza en las distinciones entre valor de uso y de no uso (Epstein citado por Mejía, 2010; Sarmiento, 2003). El primero hace referencia a ese carácter instrumental que, en ocasiones, adquieren los atributos de la naturaleza, y que les hacen ser cosas útiles: las personas utilizan los bienes ambientales, y se ven afectadas, por tanto, por cualquier cambio que ocurra con respecto a la calidad, existencia o accesibilidad de los mismos. El segundo hace referencia a los atributos de un bien que no está siendo utilizado ni que se pretende hacerlo en el futuro (Azqueta, Alviar, Domínguez y O’Ryan, 2007).
Desde el punto de vista ambiental, los bienes tiene tanto valor de uso como de no uso (Freeman, 2003, Gorfinkiel, 1999). El primero es ineludible, dadas la condición de proveedor del medio ambiente para todos los procesos empresariales (comerciales, de servicios e industriales, principalmente éstos últimos). Para el segundo, también es legítimo frente a la naturaleza, dado que los seres humanos son capaces de valorar la existencia de los recursos naturales por motivos como la benevolencia, simpatía, legado a generaciones futuras y valor simbólico (Azqueta et al., 2007; Gorfinkiel, 1999).

En este orden de ideas, la necesidad de valorar la calidad ambiental surge porque los bienes y recursos de la naturaleza son susceptibles de valorarse (Sarmiento, 2003; Costanza, 1997; Reynaldo, 2012; Abson \& Termansen, 2011; Gómez, 2005; Gorfinkiel, 1999; Mendonca \& Tilton, 2000; Rojas, Pérez y Peña, 2001), incluso para aquellos servicios no mercantiles como la depuración del agua y del aire (Westman, Odum \& de Groot et al. citados por Gómez-Baggethun, 2011), aunque la valoración sólo busca la satisfacción de necesidades informativas de la esfera empresarial (económica), dejando de lado la intención última: crear conciencia en el hombre del valor de la naturaleza para que asuma una posición de sustentabilidad con la misma, y se incluyan los resultados de investigaciones relacionadas en la toma de decisiones (Costanza et al., 1997; Machado, 2004; Hanemann, 1994). Asimismo, dicha valoración pretende la eficiencia económica al convertirse en un instrumento de política medioambiental. Incluso,

La valoración económica de los servicios del ecosistema es una herramienta política clave para detener la pérdida de diversidad biológica. Se propone que la pérdida de la 
función del ecosistema y de los recursos biológicos en los ecosistemas se debe en parte al fracaso de los mercados para reconocer los beneficios que los humanos obtienen de los ecosistemas. La asignación de valores monetarios a los servicios del ecosistema a menudo es recomendada como un paso necesario para la corrección de tales fallas del mercado. (Abson \& Termansen, 2011).

Según Barzev citado por Carbal (2009), dos hipótesis están implícitas en la valoración económica del medio ambiente:

- Las preferencias de los individuos revelan el grado de bienestar por el aprovechamiento del medio ambiente.

- Sólo los individuos determinan sus preferencias mediante su disponibilidad a pagar o a recibir.

Por otra parte, para responder al interrogante de cómo valorar la calidad ambiental, como se mencionó anteriormente, el hombre ha ideado diversos métodos, unos con más receptividad que otros, aunque en "materia de valoración de BSA no todo está dicho, por el contrario este es un terreno en el que falta mucho trabajo" (Carbal, Mantilla y Quiñones, 2010). Aún más, valorar los BSA no resulta tan sencillo, dada la inexistencia de mercados reales donde se puedan transferir la totalidad de los mismos, que incluyen algunos que se puedan tranzar y otros que no poseen tal cualidad, quedando por fuera del sistema económico (Gorfinkiel, 1999, Rojas, Pérez y Peña, 2001; Gen, 2011), y por tanto, de la esfera donde se toman las decisiones de política económica de las naciones.

Allí surge la importancia y necesidad de estudios de valoración económica de los ecosistemas, dado que tales estimaciones, al ser comparadas con los impactos generados o que se generarán por proyectos públicos o privados, permitirán realizar los análisis de costo-beneficio utilizados para incluir la dimensión económica en las decisiones de política referidas a la inversión, a la asignación de recursos y al impacto ambiental, tan necesarios en la toma de decisiones (Penna, de Prada y Cristeche, 2010; Rincón et al., 2014), función esencial de la contabilidad, aún más de su rama ambiental, que ha empezado el desarrollo de los idearios para tal fin (Geba, Fernández y Bifaretti, 2010; Jasch, 2002), fundados en una evolución epistemológica ligada a ciencias como la sociología-organizacional (Gómez, 2004; Quinche, 2008; Gómez, 2009) y su dinámica para entretejer las relaciones sociales de producción y distribución, enmarcadas a su vez en relacionamientos culturales e interacciones con la naturaleza (Ariza, 2007).

Asimismo, la contabilidad ambiental brinda información sobre la incidencia de los procesos de producción y consumo en la protección de los recursos naturales y del medio ambiente, los cuales hacen referencia esencialmente, a la tierra, el agua y la atmósfera (Machado, 2004). De allí la importancia para la contabilidad ambiental de la valoración económica de los recursos naturales.

En este sentido, a continuación se presenta el análisis de los principales métodos de valoración de la calidad ambiental.

\section{Estimación del Cambio en Productividad}

También considerado un método indirecto, busca estimar económicamente el impacto ambiental sobre un recurso natural a través de la valoración del efecto que este impacto genera en la producción, en el costo o en las ganancias generadas por otro bien que sí tiene mercado (Osorio y Correa, 2004). Por ejemplo, la calidad del agua afecta la productividad de cosechas, 
o se incurren en mayores costos para purificar el agua potable a medida que su calidad disminuye, así las ventajas económicas de mejorar la calidad del agua pueden ser medidas por el aumento de la productividad agrícola, o la disminución del costo de la purificación del agua potable (Ministerio de Ambiente, Vivienda y Desarrollo Territorial, 2003).

Para Cruz (2005), este método ha sido muy utilizado por su fácil comprensión, al basarse en la relación que existe entre el atributo ambiental y bienes y servicios existentes en el mercado; o sea que un cambio en el atributo ambiental implicará una variación en la producción del bien con que éste está relacionado, lo que afectará el bienestar o utilidad de las personas; tal variación se valora económicamente con base a precios de mercado.

El mismo autor, siguiere dos (2) etapas esenciales para la aplicación de este método:

- Determinar el efecto físico: Esta se puede dar a través de pruebas de laboratorio, resultadosdeinvestigación, experimentos controlados o técnicas de regresión estadística.

- Valorar monetariamente el efecto físico: Para valorar monetariamente el efecto físico se debe utilizar los precios de mercado, es decir, se toma una muestra representativa del área afectada y se estima las utilidades de un periodo en condiciones normales y también se estima las utilidades del periodo en condiciones en las cuales se produce un daño ambiental que afecte la producción, entonces la diferencia entre los valores presentes de las productividades físicas, multiplicado por el precio de mercado resulta el costo total en que incurre el productor por el impacto ambiental y este sería el costo ambiental (Cruz, 2005).

Este método resulta muy práctico ya que permite valorar el daño ambiental por medio de los cambios provocados en la utilidad o rentabilidad de una determinada actividad productiva que necesita de los recursos naturales para poder producir un bien canjeable en el mercado, digo que resulta muy práctico porque a través de este método ya se encuentran valorados económicamente los bienes en el mercado y solo es calcular la diferencia entre la utilidad generada cuando no existía ningún daño medio ambiental que limitara la producción con la utilidad generada cuando existe un daño que afecta directamente la producción de dicho bien.

Este método supone ciertas limitaciones que le restan efectividad. La principal sería las dificultades que se puedan presentar para establecer la relación entre el bien o atributo ambiental y el bien privado que se ve afectado por la contaminación del primero. Incluso este elemento se vuelve más desestabilizador del método si se piensa en la temporalidad de los efectos en la productividad del bien privado. Lo anterior, entraría en franca contravención del principio de objetividad de la disciplina contable.

De la misma manera, se debe considerar que los daños en el bien o atributo ambiental puede que sea causado por efectos presentes en la misma naturaleza como en el caso de elementos tóxicos presentes en el ambiente de forma natural o por acciones mismas de la naturaleza como erupciones volcánicas, tormentas o terremotos. En este caso, la valoración de la calidad ambiental debe considerar las secuelas causadas por la acción misma 
de la naturaleza, y separarlas de aquellas con origen antrópico, de tal manera que existan mecanismos para controlar y hacer seguimiento a éstas, y para hacer frente ante la presencia de aquellas.

\section{Método de Valoración Contingente}

Es el método más utilizado en el mundo, y el que cuenta con mayor aceptación (PupoGarcía y Parada-Corrales, 2015; Martínez y Roca, 2013; Ministerio de Ambiente, Vivienda y Desarrollo Territorial, 2003), lo que ha posibilitado su desarrollo científico y práctico (Rojas, Pérez y Peña, 2001). Su desarrollo surgió debido a que los métodos de preferencias reveladas eran limitados (Haab, Interis, Petrolia \& Whitehead, 2013). Incluso, ha obtenido mayor aceptación desde el estudio encargado en 1993 a un grupo de expertos por la National Oceanic and Atmospheric Administration - NOAA para evaluar el método, donde se concluyó que podría desempeñar un papel legítimo en la evaluación de daños, incluyendo la estimación de los valores de uso pasivo perdidos (Arrow citado por Haab et al., 2013), descritos por Kutrilla (1967). A lo largo de los años, el método de valoración contingente consolidó su respetabilidad siendo oficialmente reconocido por varias instituciones dedicadas a cuestiones ambientales en diversos países, lo que dio impulso a la realización de una gran cantidad de estudios que utilizaron esta metodología (Cristeche y Penna, 2008).

Es un método econométrico directo de obtención de datos por encuestas (Mejía, 2010), preguntando a los consumidores cuánto estarían dispuestos a pagar por un determinado aumento en la provisión de un bien público o servicio ambiental, o bien, cuál sería la compensación mínima que pedirían, si dicho aumento no tuviera lugar (Cano \& Cabello, 1995; Kling, Phaneuf \& Zhao, 2012). Es lo que
Martínez y Roca (2013) llaman Deposición a Pagar - DAP. También puede trabajarse mediante la DAA - Disposición a Aceptar Compensación.

Es utilizado para valorar los beneficios de una mejora ambiental de acuerdo con la cantidad monetaria que los beneficiarios potenciales de dicha mejora estarían dispuestos a pagar (DAP), o la valoración de los costos generados por un daño ambiental a través de la cantidad de dinero que los perjudicados estarían dispuestos a aceptar como compensación (DAA) por la pérdida ambiental (Riera, 1994).

Este método contempla la posibilidad de simular un mercado hipotético ${ }^{3}$ para bienes y servicios que no lo tienen, como los ambientales, al tiempo que indaga sobre las preferencias de los individuos (Kling, Phaneuf \& Zhao, 2012).

"Los supuestos teóricos de los que se sirve este método son básicamente la racionalidad del consumidor, en cuanto a su capacidad para obtener la máxima utilidad mediante el consumo de una serie de bienes dado un presupuesto, y el manejo de información perfecta por parte de quienes intervienen en un mercado". (Rojas, Pérez y Peña, 2001).

Para Gorfinkiel (1999) este método tiene ventajas y aplicaciones varias, que van desde la administración pública que necesita evaluar iniciativas para proyectos sociales, pasando por organizaciones ambientalistas preocupadas por el medio ambiente que quieren saber el valor social del patrimonio natural, hasta los tribunales que desean imponer sanciones económicas a quienes causen daños ambientales. Asimismo, permite análisis ex-ante, como el estudio planteado por Rojas, Pérez y Peña (2001), e incorpora al

3 Aunque para Gorfinkiel (1999) el mercado puede ser real 
ciudadano en la toma de decisiones, dado que es éste quién realiza la valuación del recurso y no algún experto.

Con respecto al origen de este método, Cristeche y Penna (2008) exponen que surgió en la década del setenta en los EEUU, cuando Robert Davis lo desarrolló como parte de su tesis doctoral realizando encuestas a cazadores sobre el valor que le atribuían a los bosques del estado de Maine, aunque para Hanemann (1994) Ciriacy-Wantrup lo propusieron por vez primera en 1947.

El método de valoración contingente parte esencialmente de cuestionarios aplicados a los individuos, pero tal metodología guarda ciertos vacíos que le restan efectividad a tales métodos, perdiendo objetividad (Martínez y Roca, 2013), pilar fundamental dentro de la disciplina contable, razón por la cual entran en conflicto con los supuestos teóricos de ésta (Ariza, 2007; Gómez, 2004, 2009).

En primera instancia, los cuestionarios usados en estos métodos son vulnerables a los efectos de respuesta, dado que pequeños cambios en la redacción de la pregunta o el orden de las mimas muchas veces causan cambios significativos en las respuestas de los encuestados (Longo, Hoyos y Markandya, 2015; Schuman y Presser citados por Hanemann, 1994). Asimismo, los niveles educativos de las personas también causan serios limitantes, al igual que la forma en que son abordadas por los encuestadores, y la cantidad y calidad de la información suministrada a los encuestados. Es lo que Martínez y Roca (2013) llaman sesgos de información. Incluso, el grado de conocimiento y familiaridad con el ecosistema objeto de valuación también causa variaciones significativas.
Incluso, Hanemann (1994) expone que estudios originados desde la psicología concluyen que los individuos tienden a resolver problemas de razonamiento y la elección de la forma más sencilla posible, lo que es común en la aplicación de encuestas, así como también en la psicología social, la psicología política, y estudios de mercado. Diamond, Hausman, Leonar y Denning (1993) argumentan que la gente debería preocuparse por los resultados, no sobre el proceso por el cual éstos se generan, por ende, no deberían contemplar lo que ellos piensan que es bueno para el país, en lugar de sus verdaderas preferencias económicas.

Otro limitante tiene que ver con el contenido del cuestionario, relacionado con el punto (precio) de partida, dado que a los individuos se les pide que ponga precio a algo a lo que no está habituada a hacerlo (Martínez y Roca, 2013). Finalmente, para Eberle \& Hayden (2011) otro problema potencial es la validación de los datos, dado que existen estudios empíricos que demuestran tal problemática como los realizados por Bishop y Heberlein en 1979 y Seller, Stoll y Chavas en 1985.

\section{Método de Valoración Integral}

Este se constituye en un método basado en el concepto de costo de oportunidad. Según Carbal et al. (2010) se entiende por valoración integral la cuantificación en términos monetarios de los diferentes beneficios, económicos, sociales y ambientales, que otorga la naturaleza a los seres humanos, establecidos, según precios de mercado y costo de oportunidad.

El costo de oportunidad equivale al ingreso al que renuncia quien ostenta la propiedad, posesión o dominio del área donde yacen los ecosistemas, 
garantizando la generación natural de los BSA. Este, refleja lo que deja de percibir quien tiene el dominio sobre el ecosistema, como expresión del sacrificio que asegura su disponibilidad. El valor de los BSA por costo de oportunidad, es una alternativa idónea, que se soporta en la evaluación de las potencialidades del ecosistema que los suministra, identificando su vocación técnica, a partir de sus características y haciendo uso de la racionalidad sobre los beneficios (Mantilla citado por Carbal et al., 2010).

El método de valoración integral ha sido utilizado en la valoración de los BSA ofertados por la Ciénega de la Caimanera ubicada en Coveñas, Sucre (Carbal et al., 2010) y en la Ciénaga de la Virgen, ubicada en el Cartagena, Bolívar (Carbal, Muñoz y Solar, 2014).

Este método elimina la falta de objetividad presente en los otros analizados, causada esencialmente porque la estimación del valor económico de los BSA se realiza sobre el supuesto de que la "utilización de los factores de producción, incluyendo los recursos naturales, en una forma determinada supone un costo de oportunidad equivalente al valor de uso alternativo más valioso que podría haberse destinado" (Pérez citado por Mejía, 2010). Asimismo, desecha los sesgos presentes en métodos que utilizan las preferencias de los individuos, en los instrumentos usados para obtener tales preferencias y en la determinación de relaciones causa-efecto entre los cambios de productividad de bienes mercadeables y no mercadeables, permitiendo a la disciplina contable captar la realidad social, ambiental y económica presente en las áreas donde se encuentran los ecosistemas a valorar. En otras palabras, este método potencializa la función de captación, medición y valoración razonable de los bienes y servicios ecosistémicos.

El método de valoración integral se considera como el más apropiado, a la luz de los principios de la disciplina contable, como su cercanía al postulado de medición razonable propio de la misma. Además, la estimación del valor económico de una actividad empresarial que se pueda desarrollar en un ecosistema si el mismo se destinara a tal fin, es fácilmente realizable, dado que se contarían con los insumos informativos y las herramientas económicas y financieras para tal estimación.

\section{La contabilidad ambiental como generadora de información para la toma de decisiones}

Con respecto al tercer interrogante plateado al inicio del presente paper, relacionado con la contribución de la contabilidad ambiental al debate que genera el tema de la valoración económica de los BSA, se plantean los siguientes argumentos:

Según Machado (2004), la contabilidad nacional ${ }^{4}$ brinda elementos para la planeación y ser un elemento de la política económica de un país, y un saber estratégico en el desarrollo de una nación (Mejía y Montes, 2009) que se condensa en el Sistema de Cuentas Nacionales, y dentro de éstas, el Sistema de Cuentas Satélite Ambiental (CSA), donde estadísticamente se mide en unidades físicas y monetarias, para cada período contable, la variación de los stocks de los activos ambientales, las inte-

\footnotetext{
4 Esta es una rama de la contabilidad social macro, definida en el Sistema de Cuentas Nacionales de las Naciones Unidas como "... descripción global que tiende a representar el conjunto de los fenómenos económicos en un cuadro contable coherente" (Machado, 2004).
} 
racciones entre el ambiente y la economía, dentro de la economía y de la economía al ambiente (DANE, 2014). A nivel micro, la contabilidad ambiental permite medir, analizar, evaluar y controlar la utilización de los recursos naturales y el manejo del ambiente (Machado, 2004).

Bajo estos argumentos, la contabilidad ambiental debe valerse de todas las herramientas disponibles para cumplir cabalmente con sus funciones, entre ellas, ser generadora de información oportuna, fiable, clara, objetiva y verificable, utilizada en la toma de decisiones (Alturo, 2014) a nivel macro y micro. Dicha información, que puede estar expresada en unidades monetarias (contabilidad ambiental financiera) $\mathrm{u}$ otros sistemas de medida como unidades físicas (contabilidad ambiental no financiera) (Ortega, 2007), puede ser obtenida y validada con los postulados originados en otras ciencias como la Economía y sus métodos de valoración económica ambiental. En este sentido, la contabilidad ambiental toma los insumos generados por dichos métodos, que para el caso sería el Valor Económico Total de un ecosistema en particular, para ofrecer análisis de costos ambientales de proyectos futuros. Esto se debe a la orientación de la contabilidad hacia lo que Mejía et al. (2009) refieren como un programa de investigación científico éticosocio-humanístico, que esté constituido por el deber ser en una línea naturalezahombre-economía, propia de la disciplina contable ambiental.

Con base en tal análisis, se podrán tomar los caminos adecuados para la protección y conservación de ecosistemas que serán afectados por el desarrollo antrópico, como el caso de la construcción de carreteras, muelles, embalses, hidroeléctricas, entre otros. Asimismo, la contabilidad ambiental se convierte en una herramienta de control de manejo ambiental para evaluar el impacto generado (Gómez, 2008), permitiendo comparar periódicamente los hechos económicos, con el fin de definir los avances en la responsabilidad social de las empresas y en la sostenibilidad (Alturo, 2014).

Igualmente, Bañegil citado por Ariza (2007) expone que incluso la contabilidad ambiental tiene un enfoque de direccionamiento estratégico, donde capta e interpreta información suficiente, relevante y oportuna para apoyar con criterios medioambientales, la selección de estrategias producto/mercado, la determinación de inversiones, la planificación, la ejecución y el control estratégico de las decisiones empresariales.

\section{REFLEXIONES Y CONSIDERACIONES FINALES}

La principal conclusión del trabajo realizado se relaciona con la proximidad de los postulados de la disciplina contable ambiental con el método de valoración integral, dada la metodología de este último, su fundamentación en el costo de oportunidad, que está presente dentro de las ciencias económicas y la eliminación de los sesgos y críticas que presentan los otros métodos analizados.

Asimismo, se expone el por qué los BSA son susceptibles de valorarse económicamente frente a los imperativos deteoría económica. En este orden deideas, basados en los esfuerzos de los académicos en todo el mundo por abordar la temática analizada, se evidencia que la misma goza de la atención de la comunidad científica y que genera discusiones enriquecedoras sobre la aplicabilidad y aceptación de los métodos propuestos desde diferentes 
enfoques. Esto también justifica el porqué del abordaje de la mencionada temática por parte de los autores en el presente paper.

Por otra parte, la contabilidad ambiental, que apenas alcanza grandes desarrollos científicos, entra a jugar un papel estratégico y dinámico dentro de la dinámica de toma de decisiones a nivel micro y macro de la economía. La función esencial de ser generadora de información útil para la toma de decisiones, heredada de la contabilidad tradicional o financiera, se hace más imperiosa dentro de una economía que cada día necesita más del medio ambiente y donde las decisiones locales que afectan los ecosistemas tienen repercusiones nacionales, e incluso globales.

En este sentido, y aunque resulte contradictorio, establecer un valor económico para los BSA se convierte en el vehículo más consistente y certero para crear conciencia en el hombre sobre la importancia de estos para su supervivencia y permanencia en este mundo, y fundamentalmente para la toma de decisiones de forma holística. Asimismo, debe existir un acompañamiento de una fuerte dosis de educación ambiental, que a futuro se traduzca en una cultura ambiental, lo que redundará en una población más dispuesta a ejercer el control social necesario, que provoque reacciones firmes y convincentes frente a acciones empresariales que afecten los recursos naturales. Establecer el valor para un bien o atributo ambiental se convierte en la vía necesaria para que se hagan altos en el camino, y se piense en las generaciones futuras.

Finalmente, la contabilidad ambiental se convierte en herramienta vital en el análisis de costo-beneficio, al incorporar las mediciones y valoraciones de los costos ambientales en la dinámica de toma de decisiones, con su poder instrumental y lógico que facilita la abstracción de las relaciones naturaleza-empresa, y que finalmente permite la representación racional de las mismas a través de informes contables sintetizados y comprensibles para los usuarios de la información.

\section{REFERENCIAS BIBLIOGRÁFICAS}

Abson, D. \& Termansen, M. (2011). Valuing Ecosystem Services in Terms of Ecological Risks and Returns. Conservation Biology, 25 (2), 250-258.

Albert, T. (2002). Cómo escribir artículos científicos fácilmente. Gaceta Sanitaria, 16(4), 354-357. doi:10.1016/SO2139111(02)71935-3

Alturo, C. (2014). Grado de conocimiento y aplicabilidad de la contabilidad ambiental empresarial en el Espinal, departamento del Tolima. Revista Virtual Universidad Católica del Norte, 42, 207-220. Recuperado de http://revistavirtual.ucn.edu.co/index. php/RevistaUCN/article/view/506/1044

Anguera, T. (1986). La investigación cualitativa. Educar, (10), 23-50. Recuperado de http://diposit.ub.edu/dspace/ bitstream/2445/22330/1/68186.pdf

Ariza, E. (2007). Luces y sombras en el "poder constitutivo de la contabilidad ambiental". Revista Facultad de Ciencias Económicas: Investigación y Reflexión, 15(2), 45-60. Recuperado de http://www.redalyc.org/ pdf/909/90915204.pdf

Avanzas, P., Bayes-Genis, A., Pérez, L., Sanchis, J. y Heras, M. (2011). Ethical Considerations in the Publication of Scientific Articles. Revista Española de Cardiología, 64(5), 427429. doi: 10.1016/j.rec.2011.02.005

Azqueta, D., Alviar, M., Domínguez L., y O'Ryan, R. (2007). Introducción a la economía ambiental. 2da edición. McGrawHill. Madrid, España. 
Bobenrieth, M. (1994). El artículo científico original. Estructura, estilo y lectura crítica. Granada: Escuela Andaluza de Salud Pública.

Bobenrieth, M. (2002). Normas para revisión de artículos originales en Ciencias de la Salud. International Journal of Clinical and Health Psychology, 2(3), 509-523. Recuperado de http://www.aepc.es/ijchp/articulos_pdf/ ijchp-54.pdf

Boyer, T. (2014). Is a bird in the hand worth two in the bush? Or, whether scientists should publish intermediate results. Synthese, 191(1), 17-35. doi: 10.1007/s11229-012-0242-4

Cano, A. \& Cabello, J. (1995). La valoración e internalización de los costes ambientales. Cuadernos de Ciencias Económicas y Empresariales, (29), 57-86.

Carbal, A. (2009). La valoración económica de bienes y servicios ambientales como herramienta estratégica para la conservación y uso sostenible de los ecosistemas: "Caso Ciénaga La Caimanera, Coveñas - Sucre, Colombia", Criterio Libre, 7 (10), 71-89.

Carbal, A., Mantilla, E. y Quiñones, E. (2010). Valoración monetaria de los bienes y servicios ambientales ofertados por la Ciénaga La Caimanera-Colombia. Revista Saber, ciencia y libertad, 5(2), 109-130.

Carbal, A., Muñoz, J. y Solar, L. (2014). Valoración económica integral de los bienes y servicios ambientales ofertados por el ecosistema de manglar ubicado en la Ciénaga de la Virgen. Cartagena-Colombia. Manuscrito en preparación.

Corral, M. y Encinas, J. (2013). Cómo escribir un artículo. Cuestiones formales. Radiología, 55(1), 17-27. doi: 10.1016/j.rx.2013.01.002

Costanza, R., d'Arge, R., de Groot, R., Farber, S., Grasso, M., Hannon, B,... Van den Belt, M. (1997). The value of the world's ecosystem services and natural capital. Special Issue: The value of ecosystem services, 25(1), 253260. Recuperado de http://www.esd.ornl. gov/benefits_conference/nature_paper.pdf
Cristeche y Penna (2008). Métodos de valoración económica de los servicios ambientales. Recuperado de http://inta.gob. ar/documentos/metodos-de-valoracioneconomica-de-los-servicios-ambientales/at_ multi_download/file/metodos_doc_03.pdf

Cruz, G. (2005). Métodos de evaluación ambiental. En Economía aplicada a la valoración de impactos ambientales: (123 154). Primera edición. Editorial Universidad de Caldas.

Dekanski, A. (2014). How to present and publish research results. Journal of the Serbian Chemical Society, 79(12), 1561-1570. doi: 10.2298/JSC140610066D

Departamento Administrativo Nacional de Estadística - DANE. (2014). Cuenta Satélite. BogotáD.C.,Colombia. Recuperadodehttp:// www.dane.gov.co/index.php/es/cuentaseconomicas/cuentas-satelite/101-cuentasnacionales/cuentas-ambientales/2800cuentas-economico-ambientales

Diamond, P., Hausman, J., Leonar, G. \& Denning, M. (1993). Does Contingent Valuation Measure Preferences? Experimental Evidence. En J. Hausman (Ed.), Contingent Valuation: A Critical Assessment (41-89). New York: North-Holland. Recuperado de https://books. google.com.co/books?id=xuz6S2KV-kcC\&pg= PA41\&lpg=PA41\&dq=Does + Contingent + Val uation+Measure+Preferences?+Experimenta $1+$ Evidence.\&source $=$ bl\&ots $=$ Nw02alGZOP\&s ig=oUxQ8ksPKoxlw28f2Ub_0T4j2iQ\&hl=es$419 \& s a=X \&$ ei $=8 \operatorname{lrCVK}$ KSFOSAsQT mjYKwCA\&ved=0CEQQ6AEwBA\# $\mathrm{v}=$ onepage $\& \mathrm{q}=$ Does\%20 Contingent $\% 20 \mathrm{Valuation} \% 20 \mathrm{Measure} \% 20$ Preferences\%3F\%20Experimental\%20 Evidence. $\& \mathrm{f}=$ false

Eberle, D. \& Hayden, G. (2011). Crítica de la valoración contingente y del coste del viaje como métodos para la evaluación de los recursos naturales y los ecosistemas. En F. Aguilera y V. Alcántara (Ed.), De la Economía Ambiental a la Economía Ecológica (121150). Edición electrónica: CIP-Ecosocia. Recuperado de http://www.fuhem.es/ media/ecosocial/File/Actualidad/2011/ Eberle\%20y\%20Hayden.pdf 
Ezzine, D., Rico, L., Ruiz, M. \& Maris, V. (2011). The role of biodiversity in Payments for Environmental Services: disentangling the inextricable. Revista Española de Estudios Agrosociales y Pesqueros, (228), 139-163. Recuperado de http://eds.b.ebscohost.com/ eds/detail/detail?sid=7ee1fc25-d5ae-41f898bd-6ca263deab7e\%40sessionmgr112\&vi $\mathrm{d}=0$ \&hid=122\&bdata $=$ Jmxhbmc9ZXMmc21 0ZT11ZHMtbGl2ZQ\%3d\%3d\#db=edsagr\&A $\mathrm{N}=$ edsagr.ES2011000541

Freeman, M. (2003). The Measurement of Environmental and Resource Values: Theory and Methods. Washington DC, United States of America: Resources for the Future.

Geba, N., Fernández, L. y Bifaretti, M. (2010). Marco conceptual para la especialidad contable socio-ambiental. Actualidad Contable FACES, 13(20), 49-60.

Gen, S. (2011). The Dilemma of Environmental Valuation: Ethics and U.S. Policy. International Journal of the Humanities, 9(2), 134-145. Recuperado de http://ezproxy. unicartagena.edu.co:2127/eds/pdfviewer/ pdfviewer?sid=57827e63-f4c0-4c67-a896e6d09f4a6d35\%40sessionmgr110\&vid=3\& hid=117

Gómez, G. (2005). Análisis económico de las funciones ambientales del manglar en el ecosistema Sabana Camagüey. Aplicaciones sobre prospectiva y valoración económico ambiental (pp. 87-104). La Habana, Cuba: Editorial Academia. Recuperado de http:// www.bibliociencias.cu/gsdl/collect/libros/ index/assoc/HASHed7b.dir/doc.pdf

Gómez, M. (2004). Avances de la contabilidad medioambiental empresarial: Evaluación y posturas críticas. Revista Internacional Legis de Contabilidad \& Auditoría, 18, 87-119.

Gómez, M. (2009). Tensiones, posibilidades y riesgos de la contabilidad medioambiental empresarial. Contaduría Universidad de Antioquia, 54, 55-78. Recuperado de http:// aprendeenlinea.udea.edu.co/revistas/index. php/cont/article/viewFile/7963/7461

Gómez-Baggethun, E. (2011). Payments for ecosystem services from gestation in economics to implementation in environmental policy: a critical analysis. Revista Española de Estudios Agrosociales y Pesqueros, (228), 33-54. Recuperado de http://eds.b.ebscohost.com/eds/detail/ detail?sid=6569095f-4c2b-444e-8ea6-d9f6c a854845\%40sessionmgr198\&vid=0\&hid=1 22\&bdata=Jmxhbmc9ZXMmc210ZT1lZHM tbGl2ZQ\%3d\%3d\#db=edsagr\&AN=edsagr. ES2011000537

Gorfinkiel, D. (1999). La valoración económica de los bienes ambientales: una aproximación desde la teoría y la práctica. Tesis Magíster. Montevideo: Universidad de la República. Recuperado de http://decon.edu.uy/publica/ MAESTRIA/Tesis\%20Gorfinkiel.pdf

Haab, T., Interis, M., Petrolia, D. \& Whitehead, J. (2013). From Hopeless to Curious? Thoughts on Hausman's "Dubious to Hopeless" Critique of Contingent Valuation. Applied Economic Perspectives and Policy, 35(4), 593- 612. doi:10.1093/aepp/ppt029

Hanemann, M. (1994). Valuing the Environment through Contingent Valuation. Journal of Economic Perspectives, 8(4), 19-43. doi: 10.1257/jep.8.4.19

Jasch, C. (2002). Contabilidad de gestión ambiental: Principios y procedimientos (Graciela Scavone, trad.). Obra publicada originalmente en inglés.

Kling, C., Phaneuf, D. \& Zhao, J. (2012). From Exxon to BP: Has Some Number Become Better Than No Number? Journal of Economic Perspectives, 26(4), 3-26. doi: 10.1257/jep.26.4.3

Krutilla, J. (1967). Conservation Reconsidered. American Economic Review, 57(4), 777 -786. Recuperado de http://www.colorado. edu/economics/vjcourses/resource/ krutilla.pdf

Longo, A., Hoyos, D. y Markandya, A. (2015). Sequence Effects in the Valuation of Multiple Environmental Programs Using the Contingent Valuation Method. Land Economics, 91(1), 20-35. Recuperado de http://ezproxy.unicartagena.edu.co:2127/ eds/pdfviewer/pdfviewer?sid=dbefd $4 \mathrm{fe}$ ec3f-4097-a20c-0405748440a0\%40session mgr111\&vid=2\&hid=117 
Machado, M. (2004) Dimensiones de la contabilidad social. En: Revista Internacional Legis de Contabilidad y Auditoría, (17), 173-217.

Martínez, J. y Roca, J. (2013). Economía ecológica y política ambiental. Ciudad de México, México: Fondo de Cultura Económica.

Mejía, E. (2010). Contabilidad Ambiental Crítica al Modelo de Contabilidad Financiera. Recuperado de http://www.eutimiomejia. com/images/Libro_Contabilidad_ Ambiental_-_Cr\%C3\%ADtica_al_modelo_ contable_financiero.pdf

Mejía, E. y Montes, C. (2009). Enfoque crítico de la orientación socio-ambiental en la profesión contable. Revista internacional Legis de contabilidad y auditoría, (39), 149-178. Recuperado de http://www. eutimiomejia.com/articulos/enfoquecritico orientacionsocioambientalprofesion contable.pdf

Mendonca, A. \& Tilton, J. (2000). A Contingent Valuation Study of the Environmental Costs of Mining in the Brazilian Amazon. Minerals \& Energy, 15(4), 21-32. Recuperado de http:// ezproxy.unicartagena.edu.co:2150/ehost/ pdfviewer $/$ pdfviewer?sid=2232093a-7fda41e3-9a19-f620c7c88735\%40sessionmgr11 $5 \&$ vid $=0 \&$ hid $=102$

Ministerio de Ambiente, Vivienda y Desarrollo Territorial, (2003). Guía metodológica para la valoración económica de bienes, servicios ambientales y recursos naturales. Recuperado de https://www.siac.gov.co/ documentos/DOC_Portal/DOC_Uso\%20 de\%20Recursos/Instrumentos $\% 20$ economicos/20111007_guiavaloracion $\% 20$ MAVDT\%202003.pdf

Moreno, F., Marthe N. y Rebolledo, L. (2010). Cómo escribir textos académicos según normas internacionales. Barranquilla, Colombia: Editorial Universidad de Norte. Norena, A., Alcaraz, N., Rojas, J. y Rebolledo, D. (2012). Aplicabilidad de los criterios de rigor y éticos en la investigación cualitativa. Aquichan, 12(3), 263-274. Recuperado de http://ezproxy.unicartagena.edu.co:2128/ eds/pdfviewer/pdfviewer?sid=6bfe4eb56f20-41d9-8ca2-9f1c56cbff15\%40sessionmg $\mathrm{r} 4003 \&$ vid $=3 \&$ hid $=4102$
Ortega, F. (2007). Contabilidad ambiental en términos no monetarios. En A. Carbal (Ed), Memorias del XVII Congreso Colombiano de Contadores Públicos y Encuentro Contable Latinoamericano (pp. 109-124). Medellín, Colombia: Lealon.

Osorio, J. y Correa, F. (2004). Valoración económica de costos ambientales: Marco conceptual y métodos de estimación. Semestre económico, 7(13), 159-193. Recuperado de http://www.redalyc.org/articulo. oa?id=165013657006

Páramo, P., Ortega, X. y Rodríguez, L. (2008). Aspectos éticos en la investigación social. En: Investigación en las ciencias sociales. Bogotá, Colombia: Universidad Piloto de Colombia.

Penna, J., de Prada, J. y Cristeche, E. (2010). Valoración económica de los servicios ambientales: teoría, métodos y aplicaciones. En P. Laterra, E. Jobbagy y J. Paruelo. Valoración de Servicios Ecosistémicos. Conceptos, herramientas y aplicaciones para el ordenamiento territorial, (85-119). Recuperado de http://www.iai.int/files/ LaterraJobbagyParueloValorEcosyst.pdf

Pupo-García, L. C., \& Parada-Corrales, J. (2015). Valoración económica de los bienes y servicios ecosistémicos del golfo de TribugáColombia. Revista Panorama Económico, 23(1), 39-54.

Quinche, F. (2008). Una evaluación crítica de la contabilidad ambiental empresarial. Revista Facultad de Ciencias Económicas: Investigación y Reflexión, 16(1). Recuperado de http://www.scielo.org.co/scielo.php?pi$\mathrm{d}=$ S0121-68052008000100014\&script $=\mathrm{s}-$ ci_arttext

Reynaldo, C. (2012). La Economía Ambiental y su Evolución en el Pensamiento Económico. Recuperado de http://www. eumed.net/rev/delos/13/clra.html Riera, P. (1994). Manual de valoración contingente. Recuperado de http://www. cepal.org/ilpes/noticias/paginas/0/35060/ Manual_Evaluacion_Contingente.pdf

Rincón, A., Echeverry, M., Piñeros, A., Tapia, C., David, A., Arias, P. y Zuluaga, P. (2014). Valoración integral de la biodiversidad 
y los servicios ecosistémicos: Aspectos conceptuales y metodológicos. Instituto de Investigación de Recursos Biológicos Alexander von Humboldt (IAvH). Bogotá, D. C. Colombia.

Rojas, J., Pérez, M. y Peña, M. (2001). La valoración contingente: una alternativa para determinar la viabilidad financiera de proyectos de tratamiento de aguas residuales en zonas rurales de países tropicales. Universidad del Valle Instituto CINARA, 1-14.

Sarmiento, M. (2003). Desarrollo de un nuevo modelo de valoración medio ambiental. Tesis Doctoral. Madrid: Universidad Politécnica de Madrid. Recuperado de http://oa.upm.es/105/1/07200318.pdf
Smith, A. (1776). La Riqueza de las Naciones. Recuperado de https://www.marxists.org/ espanol/smith_adam/1776/riqueza/smithtomo1.pdf

Vergara-Serpa, B., Navarro, J. L., \& Cifuentes, G. (1996). Métodos de valoración económico ambiental aplicados a la incidencia del Canal del Dique en la bahía de Cartagena, un ejercicio preliminar. Revista Panorama Económico, 4(4), 48-53.

Vergara-Arrieta, J., \& Álvarez-Carval, Y. (2016). Una mirada a los métodos de valoración de calidad ambiental desde una visión contable ambiental. Panorama Económico, 24, 221-238.

\section{AUTORES}

José Julio Vergara-Arrieta

Magíster en Administración de la Universidad de Medellín. Especialista en Finanzas y Contador Público Universidad de Cartagena. Actualmente es Docente/Investigador de la Facultad de Economía y Negocios de la Universidad Tecnológica de Bolívar. Autor de varias publicaciones en revistas indexadas y ponente en eventos académicos internacionales.

\section{Yesith Álvarez-Carval}

Administración Industrial. Investigador activo del Grupo de Investigación GIDEA adscrito al programa de Contaduría Pública de la Universidad de Cartagena. 
JOSÉ JULIO VERGARA-ARRIETA, YESITH ÁLVAREZ-CARVAL

Una mirada a los métodos de valoración de calidad ambiental desde una visión contable ambiental 